\title{
Study on Cold Chain Transportation Model of Fruit and Vegetable Fresh-Keeping in Low-Temperature Cold Storage Environment
}

\author{
Xiaolong Jiao $\mathbb{D}$, Wen Xu, and Lintong Duan \\ Hebei Vocational University of Industry and Technology, Shijiazhuang 050000, China \\ Correspondence should be addressed to Lintong Duan; 348332386@qq.com
}

Received 19 October 2021; Accepted 23 November 2021; Published 18 December 2021

Academic Editor: Daqing Gong

Copyright $\odot 2021$ Xiaolong Jiao et al. This is an open access article distributed under the Creative Commons Attribution License, which permits unrestricted use, distribution, and reproduction in any medium, provided the original work is properly cited.

\begin{abstract}
Due to the limitation of later stage intelligent algorithms, the fruit and vegetable fresh-keeping cold chain transportation scheme did not meet the expectation and could not achieve the dual objectives of the shortest time and the lowest consumption at the same time. In order to solve the above problems, a cold chain transportation model of fruit and vegetable fresh-keeping in a lowtemperature cold storage environment is proposed. The model is based on the topology of the cold chain transportation network. By setting the assumptions of the fruit and vegetable fresh-keeping cold chain transportation model, the objective model is composed of three parts: vehicle power fuel consumption cost, cold chain transportation refrigeration cost, and total fruit and vegetable loss cost. Under six constraints, the improved ant colony algorithm is used to find the optimal fruit and vegetable freshkeeping cold chain transportation route. The experimental results show that compared with the methods based on ALNS, genetic algorithm, and quantum bacterial foraging optimization algorithm, the research method can bring the best comprehensive benefit by accomplishing the fruit and vegetable transportation task in the shortest time at the lowest cost, and the research goal is thus achieved.
\end{abstract}

\section{Introduction}

Fruit and vegetable consumption takes up a large part of our daily consumption. Different from other types of food, fruits and vegetables have very short shelf life because of high water content and vulnerability, and they easily go rotten, especially in a high-temperature environment such as summer. According to statistics, 80 percent of fruit and vegetable loss occurs in the transportation link [1]. In such a context, the transportation environment should meet high standards in order to guarantee the freshness of fruits and vegetables. Cold chain transportation, as the most frequently used fruit and vegetable transportation method nowadays, refers to the transportation of food at a preset low temperature to guarantee the freshness of food during transportation to reduce food loss. However, a low-temperature environment is maintained through vast energy consumption, which means that cold chain transportation consumes a large amount of energy. Thus, the delivery cost is greatly raised [2]. As mentioned above, the optimization of the transportation route becomes especially important.
Optimized transportation route planning can greatly shorten the transportation time so as not only to reduce fruit and vegetable loss but also to lower logistics transportation cost. Many experts and scholars at home and abroad have done a relevant study on the planning of transportation routes. For example, Theeraviriya et al., targeting fuel consumption and taking the physical condition of roads as constraints, came up with the adaptive large neighborhood search (ALNS) model based on the heuristic method to solve location decision-making and transportation route planning problems [3]. Shrivastava et al. studied the distribution and inventory planning in a supply chain under transportation route disruptions and uncertain demands where, when faced with different amounts of damaged goods supply, enterprises need to make suitable distribution strategies and optimal inventory planning strategies at the risk of disruptions to maximize expected profits [4]. Yang considered the dynamic uncertainty of the urban traffic network based on distribution route optimization and planned an optimized distribution route utilizing the objective function of minimum cost and employing genetic algorithms for 
solutions [5]. Ning et al. proposed the optimization method of the cold chain distribution route of fresh agricultural products under carbon tax mechanism in allusion to the heavy carbon emission during fresh agricultural products cold chain distribution. Targeting minimum carbon emission cost and comprehensive cost, this method introduced the carbon tax mechanism; conducted quantitative analysis; adopted constraints of quantity demanded, time demanded, and discharge time; calculated the optimal solution of the distribution model using quantum bacterial foraging algorithm; and then obtained the shortest distribution route [6].

Despite certain achievements through the application of the previous methods, the transportation schemes obtained did not meet expectations affected by later stage solutions. Therefore, a cold chain transportation model of fruit and vegetable fresh-keeping in a low-temperature cold storage environment is built in combination with previous research experiences in allusion to the problems mentioned above. The model is expected to provide a reliable basis for cold chain transportation planning.

\section{Materials and Methods}

As ready-to-eat products, fresh fruits and vegetables are not easy to preserve and have a short shelf life. Therefore, cold chain transportation becomes the key to guaranteeing the freshness of fruits and vegetables. In cold chain transportation, fruits and vegetables are preserved in removable cold storages to maintain a constant low-temperature environment to effectively guarantee the freshness of fruits and vegetables. However, the maintenance of a long-time lowtemperature environment consumes a large amount of energy and thus increases the transportation cost [7]. In such a context, effective planning of the formulation of transportation schemes becomes crucial. Optimal transportation routes can greatly shorten the transportation time, which means not only less fruit and vegetable loss and better freshness but also less energy consumption and lower transportation cost. Based on the aforementioned analysis, the problem of fruit and vegetable fresh-keeping cold chain transportation can be reduced to the problem of optimization of vehicle routes and transportation routes [8]. The research mainly contains the following parts, namely, the analysis of cold chain transportation network topology, problem description and model assumption, the establishment of target models, the setting of constraints, and optimal solutions of models. The following are detailed analyses of these parts.

\subsection{Analysis of Cold Chain Transportation Network Topology.} Cold chain transportation network topology refers to the transportation network composed of the distribution center and all customer points, as shown in Figure 1.

Figure 1 shows the basic topology of the cold chain transportation network, composed of one center point and multiple customer points. Nevertheless, a single transportation center network cannot meet demands in terms of large regions and big populations; therefore, one region usually has multiple center points and more customer points [9]. With each center point in charge of the transportation and distribution of a portion of customer points, a large network is formed with multiple distribution centers.

The determination of the distribution of center points and multiple customer points and the determination of the cold chain transportation network topology in one region are the precondition and foundation for transportation route planning [10]. GIS (Geographic Information System) is employed here to build the transportation network map which contains three parts: the construction of the road network, the point mapping, and the description of the transportation network map. The detailed analyses are as follows.

2.1.1. Construction of Road Network. The road network means the routes of connection between the points in one region [11], and the construction process is as follows:

Step 1: To output the basic information of each route to form Set $E$. The basic information of a route includes road name, route code, road grade, road length, road width, and road condition factor.

Step 2: To determine the cross point and isolated endpoint to form Set $V$. In Set $V$, each point or endpoint is given its attribute information, that is, the serial numbers of crossroads and the coordinates of cross points and isolated endpoints.

Step 3: To draw the electronic map of the road network.

2.1.2. Point Mapping. Point mapping is to map the center points and customer points and label them on the electronic map of the road network according to their coordinates [12]. The detailed process is as follows:

Step 1: To input the information of the points to form Set $C$. The information of the points includes the point code, the point attribute, and demanded quantity of points.

Step 2: To map the points onto the electronic map of the road network through vertical mapping.

Step 3: To conduct appropriate adjustment of mapping points according to the actual situation.

Step 4: To compose superposition points and the road network and store the mapping points to form Set $C^{\prime}$. Set $C^{\prime}$ includes the following information: the correspondence between the mapping points and the points in Set $C$, the coordinates of the points before and after mapping, and the serial numbers of the routes related to the points.

2.1.3. Description of the Transportation Network. Based on the above two steps, the transportation network topology is constituted and described as follows:

$$
Y=\left\{C^{\prime}, E, V, W\right\}
$$

where $W$ is the positive real number set, the weight of the route [13]. 


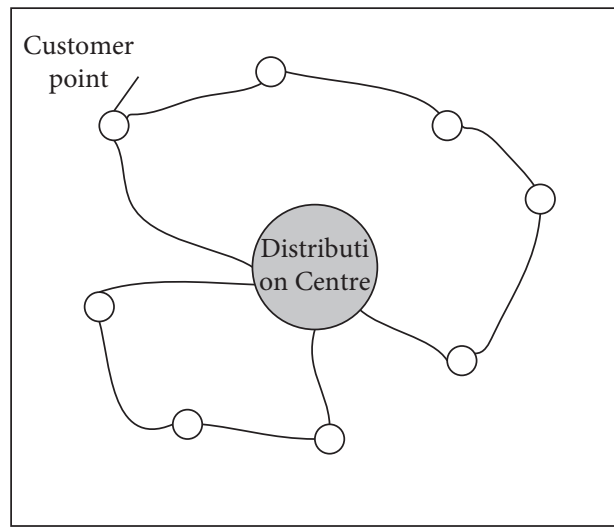

Figure 1: Basic topology of cold chain transportation network.

\subsection{Problem Description and Model Assumption}

2.2.1. Problem Description. Problem description is to conduct definition and description in allusion to the fruit and vegetable transportation route planning. The description of the problem can better pinpoint the direction and goal of research and serve as the guidance of the whole study [14]. Based on this study, that is, the fruit and vegetable freshkeeping cold chain transportation in a low-temperature environment, the description is as follows: on the basis of cold chain transportation network, all cold chain transportation vehicles depart from the distribution center point in the network and deliver fruits and vegetables to retailers in the shortest time on minimum consumption through appropriately planned transportation routes when preset constraints are met [15].

2.2.2. Model Assumption. The route planning of fruit and vegetable cold chain transportation in a low-temperature cold storage environment is subject to the effect of many factors while many of them are not major factors and have only a minor effect on route planning. Therefore, the problem study is simplified to reduce the impact of minor factors on research results [16]. Some assumptions need to be premade based on the study in the previous chapter as follows:

Assumption 1: the distribution center is the starting point, and the vehicle returns to the starting point after completing the distribution

Assumption 2: all distribution vehicles have the same load capacity and are all equipped with cold storage functions

Assumption 3: all vehicles only accomplish the delivery task without the goods consolidation function [17]

Assumption 4: the distribution center has abundant inventory, and there is no possibility of stockout

Assumption 5: fruits and vegetables need to be distributed to each and every customer point only once

Assumption 6: the low-temperature cold storage environment temperature is fixed during transportation [18]
Assumption 7: the freshness of fruits and vegetables is only affected by transportation time and their own shelf life

Assumption 8: the roads between points are clear of congestions

Assumption 9: in the simulation state, all vehicles are free from any accident during distribution

Assumption 10: the demands of each and every customer are met after the distribution task is accomplished [19]

2.3. Construction of the Objective Model. The objective model, also known as the objective function, is the description of the function of the objective to be realized, the basic model of which is as follows:

$$
\frac{\min }{\max } f=f(x)
$$

where $x$ represents the objective function value and $f(x)$ represents the objective function [20]:

$$
\min f=A+B+C \text {. }
$$

Based on the abovementioned basic model of the objective function, the cold chain transportation model of fruit and vegetable fresh-keeping in a low-temperature cold storage environment is established, and the mathematical description is as follows:

$$
\min f=A+B+C,
$$

of which,

$$
\begin{aligned}
A & =\sum_{i=1}^{N} \sum_{j=1}^{N} \sum_{l=1}^{L} \sum_{k=1}^{K} D_{i j} X_{i j}^{l k} E_{i j} \\
& =\sum_{i=1}^{N} \sum_{j=1}^{N} \sum_{l=1}^{L} \sum_{k=1}^{K} D_{i j} X_{i j}^{l k} E_{1} \cdot\left[\delta\left(E_{2}-E_{1}\right)\right],
\end{aligned}
$$

where $A$ represents the fuel consumption cost of vehicle power; $N$ represents the number of customer points; $K$ represents the number of distribution vehicles; $X_{i j}^{l k}$ represents the distribution demand, $X_{i j}^{l k}=1$ when there is demand and $X_{i j}^{l k}=0$ otherwise; $D_{i j}$ represents the distance between Point $i$ and Point $j$; $E_{i j}$ represents the fuel consumption cost between Point $i$ and Point $j ; E_{1}$ and $E_{2}$ represent no-load and full-load fuel consumption costs, respectively; $\delta$ represents the full load degree coefficient [21]:

$$
B=\gamma \phi \varphi \xi\left[\sum_{l=1}^{L} \sum_{k=1}^{K} \sum_{i=1}^{N} \sum_{j=1}^{N} X_{i j}^{l k}\left(t_{i j}+t_{i}^{o}+t_{i}^{w}\right)\right],
$$

where $B$ represents the refrigeration cost of cold chain transportation; $t_{i j}$ represents the travel time spent from Point $i$ to Point $j$; $t_{i}^{o}$ represents the time when the distribution task is completed at Point $i$; $t_{i}^{w}$ represents the time the customer spends in waiting for the accomplishment of the distribution task at Point $i ; \gamma$ represents the coefficient of heat conduction of the carriage; $\phi$ represents the area of the 
cool storage of the vehicle; $\varphi$ represents the temperature difference between the inside and outside of the cool storage of the vehicle; $\xi$ represents the fuel consumption for refrigeration for accomplishing one cold chain transportation:

$$
C=\sum_{i=1}^{N} \sum_{j=1}^{N} \psi\left[\left(t_{i}^{0}+\frac{D_{i j}}{v_{i j}}\right) \lambda+W Q \varphi t_{i}^{0}\right]
$$

where $C$ represents the total cost of fruit and vegetable loss; $\psi$ represents the cost price of fruits and vegetables of 1 jin $(1 \mathrm{jin}=0.5 \mathrm{~kg}) ; \lambda$ represents the average working efficiency; $v_{i j}$ represents the average running speed of the transportation vehicle in the travel between Point $i$ and Point $j ; W$ represents the damage ratio of fruits and vegetables during transportation; $Q$ represents the maximum weight of fruits and vegetables of the transportation vehicle at one time.

The cold chain transportation model of fruit and vegetable fresh-keeping in low-temperature cold storage environment here established is a multiple objective function which is mainly composed of three parts: the fuel consumption cost of vehicle power, the refrigeration cost of cold chain transportation, and the total cost of fruit and vegetable loss [22]. The aim is to seek the optimal transportation route through the three objectives.

2.4. Setting of Constraints. Objective functions are to be solved in the scope defined by constraints [23]. Therefore, constraints should be set for the objective model established above.
(1) Constraint 1: the constraint on distribution service at customer points:

$$
\sum_{l=1}^{L} \sum_{k=1}^{K} X_{i j}^{l k}=1, \quad \forall k \in K, i \in N
$$

where $N$ represents the number of customer points.

(2) Constraint 2: the constraint on fruit and vegetable load capacity:

$$
\sum_{i=1}^{N} X_{i j}^{l k} q_{i} \leq Q, \quad \forall k \in K ; l \in L,
$$

where $q_{i}$ represents the quantity demanded at Point $i$.

(3) Constrain 3: the constraint on vehicle service: $\sum_{i=1}^{N} Y_{i j}^{l k} \leq 1, \quad \forall j \in\{1,2, \ldots, N+L\} ; k \in K ; l \in L$,

where $Y_{i j}^{l k}$ represents whether the $k$ th vehicle departing from distribution center $l$ will go by the route from Point $i$ to Point $j$; $Y_{i j}^{l k}=1$ if yes and $Y_{i j}^{l k}=$ 0 otherwise.

(4) Constraint 4: the constraint on the balance of traffic flow on all transportation routes:

$$
\sum_{i=1}^{N} X_{i j}^{l k}\left(Q_{i j}-q_{i}\right) \geq 0, \quad i, j=1,2, \ldots, N ; l=1,2, \ldots, L ; k=1,2, \ldots, K
$$

where $Q_{i j}$ represents the weight of fruits and vegetables on the vehicle on the route between Point $i$ and Point $j$.

(5) Constraint 5: the constraint on no transportation link between central warehouses:

$$
\sum_{k=1}^{K} \sum_{i=1}^{N} \sum_{j=1}^{N} q_{i} X_{i j}^{l k} \leq \zeta_{l}, \quad l=1,2,3, \ldots, L
$$

where $\zeta_{l}$ represents the volume of fruits and vegetables that can be held by central warehouse $l$.

(6) Constraint 6: the constraint on the total length of every transportation route:

$$
\sum_{i \in N} \sum_{j \in N} S_{i j} Y_{i j}^{l k} \leq \max S^{k}, \quad k \in K
$$

where $S_{i j}$ represents the distance from Point $i$ to Point $j$ and $\max S^{k}$ represents the maximum mileage of vehicle $k$.

The objective model is to be solved in the following step based on the six constraints.

2.5. Solution of the Objective Model. To solve the objective model is to seek the optimal solution that meets the objective function by utilizing intelligent optimization algorithms under the abovementioned constraints. In a previous study, the solving methods adopted in this step are mostly basic optimization algorithms. In contrast, basic optimization algorithms often have their drawbacks that prevent the solution from reaching the optimal value [24]. Therefore, the fundamental ant colony algorithm is utilized as the basis here with some improvement. To improve the ant colony algorithm, we need to know its drawbacks. The main problem of this algorithm is the incidental local optimum problem, which is also the leading cause of the failure in 
finding the optimal solution [25]. Accordingly, this study optimizes the algorithm in the following aspects:

(1) Based on the original version, the improved state transition rule adopts a random number $r$ with its value within the range of $0 \sim 1$. The improved state transition rule is as follows:

$$
P_{i j}^{k}(t)=\left\{\begin{array}{l}
\frac{r \cdot g_{i j}^{\alpha}(t)}{\sum_{s \in F} h_{i j}^{\beta}(t)}, \quad j \in F, \\
0, \quad \text { otherwise, }
\end{array}\right.
$$

where $P_{i j}^{k}(t)$ represents the probability of Ant $\mathrm{k}$ transiting from Point $i$ to Point $j$ at Moment $t ; F$ represents the set of points the ant can reach; $\alpha$ represents the information heuristic factor; $\beta$ represents the expectation heuristic factor; $g_{i j}$ represents the pheromone concentration between Point $i$ and Point $j ; h_{i j}$ represents the expectation pheromone concentration between Point $i$ and Point $j$; and $t$ represents the time. The improved state transition rule structure is as shown in Figure 2.

(2) The improved pheromone update rule:

The pheromone update rule substitutes $Z$, the length of the optimal route in the previous circulation, with $Z_{k}$ :

$$
\begin{gathered}
g_{i j}^{\prime} \longleftarrow(1-q) \cdot g_{i j}+q \Delta g_{i j}, \\
\Delta \tau_{i j}=\left\{\begin{array}{l}
\left(\frac{Q}{Z_{k}}\right), \quad i, j \in \text { abcdef }, \\
0, \quad \text { otherwise. }
\end{array}\right.
\end{gathered}
$$

$q$ represents the volatilization degree of the pheromone of the ant per unit time; $Q$ represents the initial concentration of the pheromone of the ant; $Z_{k}$ represents the set of global optimal routes; $\Delta g_{i j}$ represents the value difference of $Q$ before and after the update; $g_{i j}$ and $g_{i j}^{\prime}$ represent the pheromone concentrations on the route between Point $i$ and Point $j$ before and after the update, respectively.

The basic solution flow of transportation routes is as shown in Figure 3.

According to the solution flow based on the improved ant colony algorithm in Figure 3, the actual situation of the cold chain transportation plan is obtained, and the ant colony searching is conducted. When the preset termination conditions are met, the objective model solution is ended, the transportation result scheme is obtained, the solution of the objective function is completed, the optimal cold chain transportation route is obtained, and the cold chain transportation model of fruit and vegetable fresh-keeping in a low-temperature cold storage environment is established.

\section{Result and Analysis}

To test the application effect of the model established, a simulation experiment is conducted in comparison with the ALNS based method, genetic algorithm-based method,

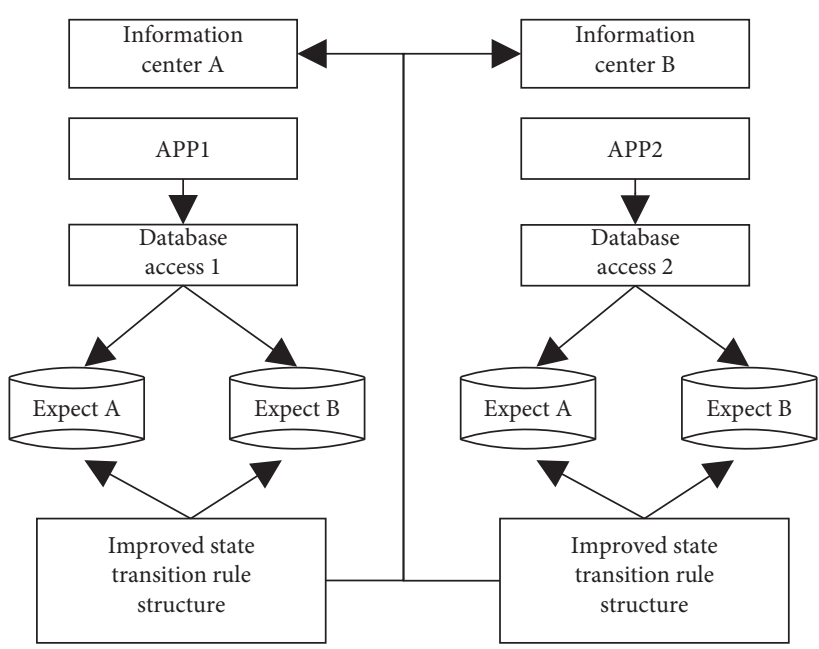

FIGURE 2: Diagram of improved state transition rule structure.

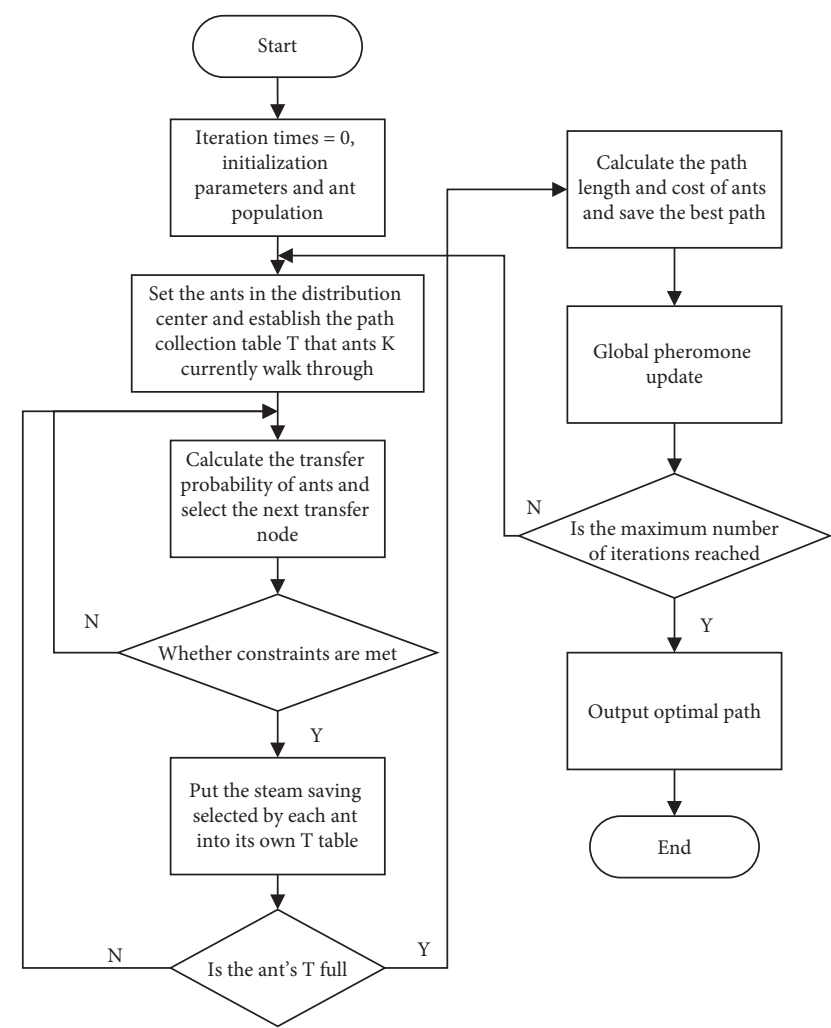

Figure 3: Solution flow based on improved ant colony algorithm.

and quantum bacterial foraging optimization algorithmbased method. In the simulation experiment, the simulation platform is Pentium $42.8 \mathrm{GHz}$ with $64 \mathrm{G}$ memory, the operating system is XP SP2, the dominant frequency of $\mathrm{CPU}$ is Intel Xeon E5620/2.4 GHz, the hard disk size is 256G, and the MATLAB tool is employed. The experiment conditions are the provision of node storage space and node digital signal processor, the satisfaction of the Ethernet controller, and being supportive of software protocols. 


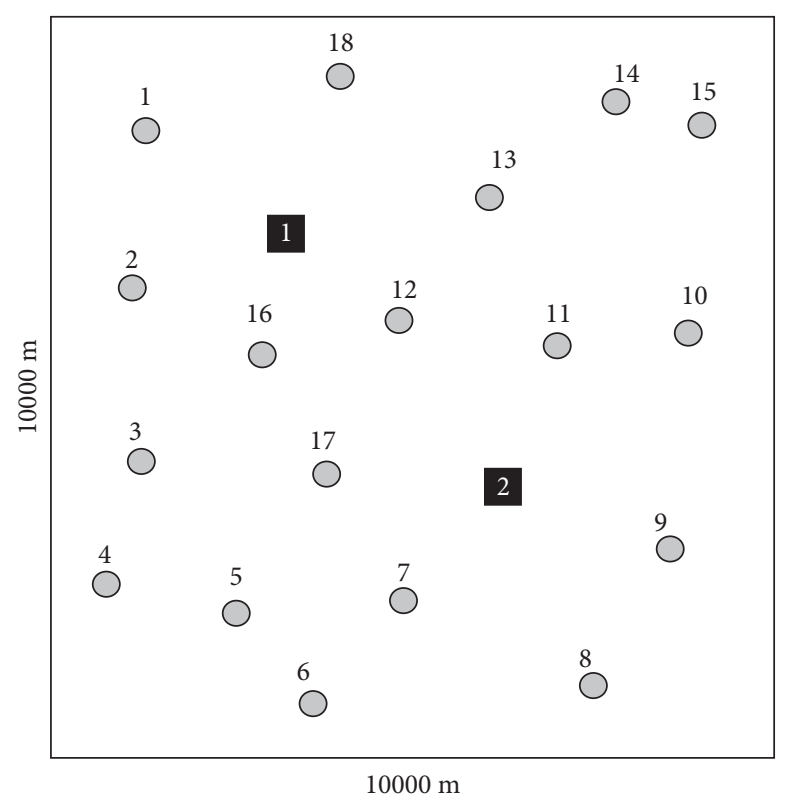

FIgURE 4: Transportation network topology.

TABle 1: Parameter setting of simulation experiment.

\begin{tabular}{lccc}
\hline Model parameter & Numerical value & Algorithm parameters & Numerical value \\
\hline Number of vehicles delivered & 2 & Number of ants (number of vehicles) \\
The maximum mileage & $100 \mathrm{~km}$ & $\alpha$ & 2 \\
The volume of the carriage & $12.7 \mathrm{~m}^{3}$ & $\beta$ & 1 \\
Vehicle speed & $30 \mathrm{~km} / \mathrm{h}$ & 2 & 0.3 \\
Average outside temperature & $21^{\circ} \mathrm{C}$ & Maximum number of iterations \\
Unit refrigerant cost & $1.5 \mathrm{yuan}$ & $r$ & 0.8 \\
Fuel consumption rate per unit time & $22.37 \mathrm{~g} / \mathrm{s}$ & - & - \\
Heat transfer derivative of refrigerator truck & 0.4 & - & - \\
Energy consumption constant & 44 & - & - \\
Transportation cost per unit mileage & 2 yuan & -
\end{tabular}

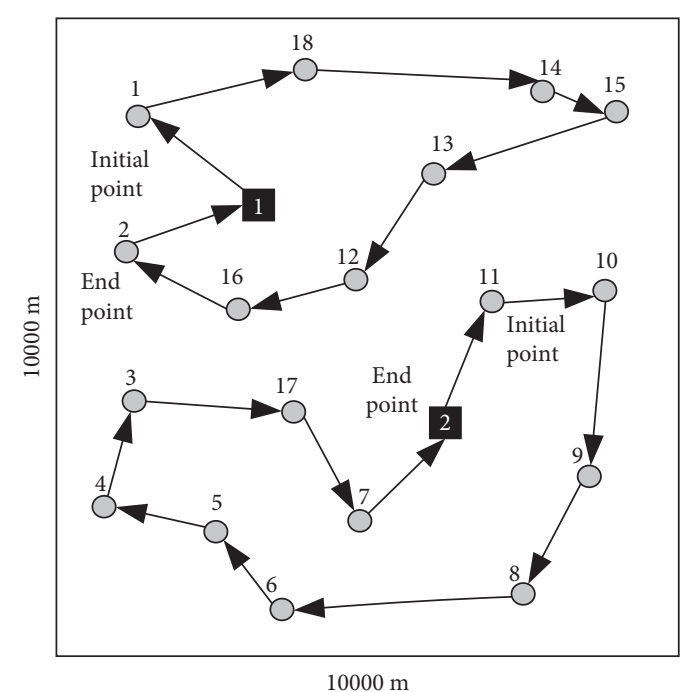

(a)

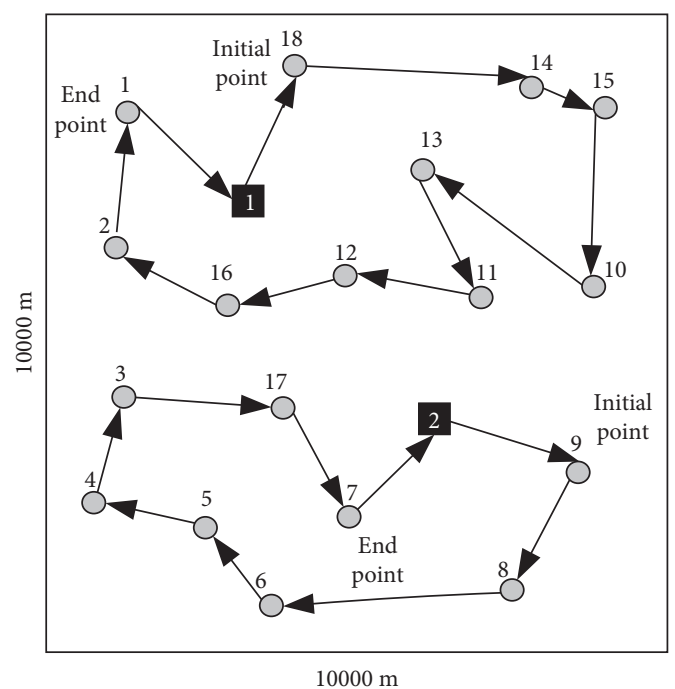

(b)

Figure 5: Continued. 


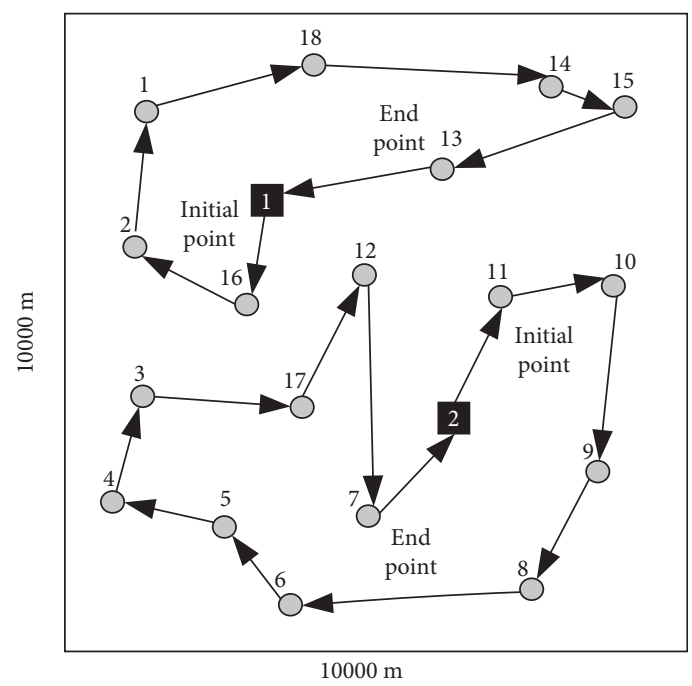

(c)

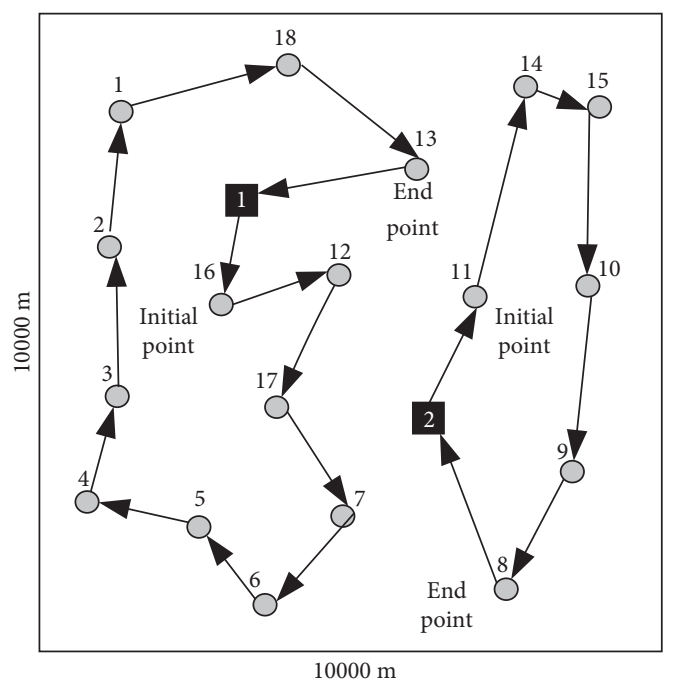

(d)

FIGURE 5: Route planning schemes. (a) Research model. (b) ALNS based method. (c) Genetic algorithm-based method. (d) Quantum bacterial foraging optimization algorithm-based method.

TABLE 2: Comparison of transportation time and various costs of four different planning schemes.

\begin{tabular}{|c|c|c|c|c|c|}
\hline Methods & $\begin{array}{l}\text { Transportation } \\
\text { time }(\mathrm{h}) \\
\end{array}$ & $\begin{array}{l}\text { Vehicle power and fuel } \\
\text { consumption cost (yuan) }\end{array}$ & $\begin{array}{l}\text { Refrigeration cost } \\
\text { (yuan) }\end{array}$ & $\begin{array}{c}\text { Loss cost of fruits and } \\
\text { vegetables (yuan) }\end{array}$ & $\begin{array}{c}\text { Total cost } \\
\text { (yuan) }\end{array}$ \\
\hline Research model & 2.54 & 354.94 & 65.44 & 187.64 & 608.02 \\
\hline ALNS based method & 2.68 & 420.05 & 77.32 & 200.36 & 697.73 \\
\hline Genetic algorithm based method & 3.20 & 435.52 & 76.23 & 214.64 & 726.39 \\
\hline $\begin{array}{l}\text { Quantum bacterial foraging } \\
\text { optimization algorithm based } \\
\text { method }\end{array}$ & 2.88 & 387.31 & 72.34 & 189.57 & 649.22 \\
\hline
\end{tabular}

\subsection{Overview of Research Area. Adopt an area of $10000 \mathrm{~m} *$} $10000 \mathrm{~m}$ as the research area to conduct the cold chain transportation route planning research, as shown in Figure 4.

In Figure 4, there are 2 distribution centers in total, each represented by a " $\square$ " and 18 customer points in total, each represented by a "O."

\subsection{Parameter Setting of the Simulation Experiment.} Parameters referred to in the simulation experiment are as shown in Table 1.

3.3. Route Planning Scheme. Conduct distribution route planning according to the research model and the three comparative methods. The route planning schemes are as shown in Figure 5.

It can be observed from Figure 5 that the routes planned through the four methods are different from each other. Then, total cost calculation is conducted according to the four different planning schemes.

3.4. Result Comparison. Do statistics of the transportation time and various costs of the four different planning schemes in Figure 5, and the result is as shown in Table 2.
It can be observed from Table 2 that, in comparison with the other three methods, the research method can bring the best comprehensive benefit by accomplishing the fruit and vegetable transportation task in the shortest time at the lowest cost, and the research goal is thus achieved.

\section{Conclusion}

To sum up, although cold chain transportation can guarantee the freshness of fruits and vegetables, the longer the transportation time goes, the higher the cost becomes. Therefore, how to complete the fastest fruit and vegetable cold chain transportation at the lowest cost becomes the focus of the study. For this purpose, the study is conducted on a cold chain transportation model for fruit and vegetable fresh-keeping in low-temperature cold storage to look for optimal transportation routes. The experimental results of the model are as follows:

(1) Result of transportation time: Time of the research method is the shortest, with that of the ALNS based method coming in the second place and that of the quantum bacterial foraging optimization algorithmbased method and that of the genetic algorithm-based method following successively. The reason is that the 
model in this paper sets constraints, and the solution of the objective function is conducted within the scope defined by the constraints, which can shorten the transportation time to a certain extent.

(2) Result of transportation cost: The transportation cost of the research method is the lowest, with that of the quantum bacterial foraging optimization algorithmbased method coming next and that of the ALNS based method and that of the genetic algorithmbased method following successively. The reason is that the cold chain transportation model of fruit and vegetable fresh-keeping in a low-temperature cold storage environment established in this paper is a multiple objective function, mainly composed of three parts: the fuel consumption cost of vehicle power, the refrigeration cost of cold chain transportation, and the total cost of fruit and vegetable loss. The optimal transportation route that meets the objectives can be obtained through the three objectives, which can save the transportation cost to a certain extent.

The above-mentioned comparison proves that the transportation route planned in the research model is preferable. However, the model established does not consider temporary order additions by customers; therefore, the study needs further improvement.

\section{Data Availability}

The data supporting this paper were taken from previously reported studies and datasets, which have been cited, and the processed data are available at CNKI. For further detailed information, the corresponding author should be contacted.

\section{Conflicts of Interest}

The authors declare that they have no conflicts of interest.

\section{Acknowledgments}

This research was supported by Factor Analysis and Research on the Differential Development of Rural E-Commerce in Hebei Province (YJYF201904).

\section{References}

[1] G. Wang, P. Ding, H. Chen, and J. Mu, "Green fresh product cost sharing contracts considering freshness-keeping effort," Soft Computing, vol. 24, no. 4, pp. 2671-2691, 2020.

[2] X. Xu and X. Zhang, "Simulation and experimental investigation of a multi-temperature insulation box with phase change materials for cold storage," Journal of Food Engineering, vol. 292, no. 109, Article ID 110286, 2020.

[3] C. Theeraviriya, R. Pitakaso, K. Sillapasa, and S. Kaewman, "Location decision making and transportation route planning considering fuel consumption," Journal of Open Innovation: Technology, Market, and Complexity, vol. 5, no. 2, p. 27, 2019.

[4] H. Shrivastava, A. T. Ernst, and M. Krishnamoorthy, "Distribution and inventory planning in a supply chain under transportation route disruptions and uncertain demands,"
International Journal of Information Systems and Supply Chain Management, vol. 12, no. 3, pp. 47-71, 2019.

[5] S. Yang, "Optimization of urban logistics distribution path under dynamic traffic network," International Core Journal of Engineering, vol. 6, no. 1, pp. 243-248, 2020.

[6] T. Ning, L. An, and X. Duan, "Optimization of cold chain distribution path of fresh agricultural products under carbon tax mechanism: a case study in China," Journal of Intelligent and Fuzzy Systems, vol. 40, no. 1, pp. 1-10, 2021.

[7] P. Bogiatzis, C. A. Rychert, and N. Harmon, "Multiple graph realizations method: improving the accuracy and the efficiency of the shortest path method through random sampling," Geophysical Journal International, vol. 227, no. 1, pp. 669-679, 2021.

[8] X. Wang, H. Li, J. Yang, C. Yang, and H. Gui, "Optimal path selection for logistics transportation based on an improved ant colony algorithm," International Journal of Embedded Systems, vol. 13, no. 2, p. 200, 2020.

[9] V. Kumar and K. Prasanth, "Weighted rendezvous planning on Q-learning based adaptive zone partition with PSO based optimal path selection," Wireless Personal Communications, vol. 110, no. 4, pp. 153-167, 2019.

[10] A. Nagy and I. Vajk, "Sequential time-optimal path-tracking algorithm for robots[J]," IEEE Transactions on Robotics, vol. 35, no. 5, pp. 1253-1259, 2019.

[11] Y. Hu and Y. Shuai, "Multimodal transportation and route optimization of vehicle logistics," Journal of Transportation Engineering and Information, vol. 017, no. 001, pp. 13-18, 2019.

[12] A. Tharwat, M. Elhoseny, A. Hassanien, T. Gabel, and A. Kumar, "Intelligent Bézier curve-based path planning model using Chaotic Particle Swarm Optimization algorithm," Cluster Computing, vol. 22, no. 4, pp. 1-22, 2019.

[13] A. V. Le, P. Veerajagadheswar, P. Thiha Kyaw, M. R. Elara, and N. H. K. Nhan, "Coverage path planning using reinforcement learning-based TSP for hTetran-a polyabolo-inspired self-reconfigurable tiling robot," Sensors, vol. 21, no. 8, p. 2577, 2021.

[14] B. Mehran, M. A. Khan, M. Mehran, H. Roh, and S. Sharma, "Case study scenarios in site selection of hazardous material facilities based on transportation preferences," Journal of Modern Transportation, vol. 27, no. 4, pp. 317-333, 2019.

[15] P. Han and X. Yang, "Big data-driven automatic generation of ship route planning in complex maritime environments," Acta Oceanologica Sinica, vol. 39, no. 8, pp. 113-120, 2020.

[16] J. Xie, S. Xiao, Y. Liang, L. Wang, and J. Fang, "A throughputaware joint vehicle route and access network selection approach based on SMDP," China Communications, vol. 17, no. 05, pp. 251-273, 2020.

[17] L. Qi, C. Qza, and D. Bl, "Cold chain transportation decision in the vaccine supply chain," European Journal of Operational Research, vol. 283, no. 1, pp. 182-195, 2020.

[18] H. Zhou, Y. Zhao, J. Fang, X. Chen, and K. Zeng, "Hybrid route recommendation with taxi and shared bicycles," Distributed and Parallel Databases, vol. 38, no. 3, pp. 563-583, 2020.

[19] X. Zhou, Z. Yu, L. Yuan, L. Wang, and C. Wu, "Measuring accessibility of healthcare facilities for populations with multiple transportation modes considering residential transportation mode choice," ISPRS International Journal of Geo-Information, vol. 9, no. 6, pp. 394-400, 2020.

[20] A. Bachu, R. Gracious, C. Gangrade, and L. Vanajakshi, "Citylevel route planning with time-dependent networks," Current Science, vol. 119, no. 4, pp. 680-690, 2020. 
[21] K. Jiang, D. Yang, C. Liu, T. Zhang, and Z. Xiao, "A flexible multi-layer map model designed for lane-level route planning in autonomous vehicles-ScienceDirect," Engineering, vol. 5, no. 2, pp. 305-318, 2019.

[22] G. Mannarini, D. Subramani, P. Lermusiaux, and N. Pinardi, "Graph-search and differential equations for time-optimal vessel route planning in dynamic ocean waves," IEEE Transactions on Intelligent Transportation Systems, vol. 21, no. 8, pp. 3581-3593, 2019.

[23] S. M. Novillo Villegas, A. Rodríguez, D. Chavez, and O. Camacho, "Path planning for mobile robots applied in the distribution of materials in an industrial environment," Systems and Information Sciences, vol. 1273, no. 1, pp. 323337, 2020.

[24] Y. Li, "The problem of independent distribution path of goods," Advances in Applied Mathematics, vol. 09, no. 3, pp. 346-348, 2020.

[25] C. Yao, "Research on logistics distribution path analysis based on artificial intelligence algorithms," International Journal of Biometrics, vol. 12, no. 1, pp. 100-105, 2020. 